\title{
Corela
}

Cognition, représentation, langage

HS-14 | 2013

Calcul du sens et contexte

\section{Pragmatiques de la vérité: sens, représentation et contexte, de G. Frege à Ch. Travis.}

\section{Bruno Ambroise}

\section{(2) OpenEdition}

\section{Journals}

Édition électronique

URL : http://journals.openedition.org/corela/3082

DOI : $10.4000 /$ corela.3082

ISSN : $1638-573 \mathrm{X}$

\section{Éditeur}

Cercle linguistique du Centre et de I'Ouest - CerLICO

Référence électronique

Bruno Ambroise, «Pragmatiques de la vérité: sens, représentation et contexte, de G. Frege à Ch. Travis. », Corela [En ligne], HS-14 | 2013, mis en ligne le 25 juin 2014, consulté le 19 avril 2019. URL: http://journals.openedition.org/corela/3082 ; DOI : 10.4000/corela.3082

Ce document a été généré automatiquement le 19 avril 2019

\section{(c) (i) (2)(2)}

Corela - cognition, représentation, langage est mis à disposition selon les termes de la licence Creative Commons Attribution - Pas d'Utilisation Commerciale - Partage dans les Mêmes Conditions 4.0 International. 


\title{
Pragmatiques de la vérité: sens, représentation et contexte, de $\mathrm{G}$. Frege à Ch. Travis.
}

\author{
Bruno Ambroise
}

1 Héritier direct des conceptions de J.L. Austin ${ }^{1}$ et de L. Wittgenstein ${ }^{2}$, Ch. Travis occupe une position singulière dans le champ philosophique, qualifiée de "contextualisme radical ». Cette position, qui a des répercussions immédiates en linguistique pragmatique ${ }^{3}$ , entend donner un rôle décisif au "contexte » en montrant que son intervention est nécessaire pour penser le fonctionnement du langage, notamment pour identifier le sens des énoncés et dire la vérité. Ce faisant, Travis s'oppose à une conception bien ancrée dans la philosophie du langage analytique, souvent attribuée à G. Frege et selon laquelle la signification d'un énoncé (voire d'un terme linguistique) permet de déterminer ses conditions de véritét. Chez Frege, la signification d'une assertion correspond ainsi à une pensée (qu'on peut identifier à une "proposition») qui, lorsqu'elle a une référence, renvoie à une valeur de vérité. Dans la tradition qui s'en inspire, l'élucidation de la signification d'un énoncé passe par la détermination de ses conditions de vérité, lesquelles permettent en retour d'identifier un sens stable qui ne varie pas d'énonciation en énonciation, c'est-à-dire selon l'usage qui est fait de cet énoncé. On parle alors traditionnellement, là encore, de la «proposition » exprimée par un énoncé, strictement corrélée à ses conditions de vérité. Or, dans son combat en faveur du contextualisme, Travis, s'il ne s'attaque pas complètement à l'idée d'une détermination possible de la signification $^{5}$, ni à celle de la vérité, s'en prend au représentationnalisme implicite dans toute théorie qui postule l'existence d'une proposition qui se situerait au-delà, ou en deçà, des usages des énoncés et qui en déterminerait uniment le sens ${ }^{6}$. Ce faisant, il entend détacher les questions de sémantique des questions de vérité : la vérité n'est pas l'affaire du (seul) sens des mots, mais une question d'usage, c'est-à-dire pragmatique ${ }^{7}$. Pour le dire autrement, ce n'est pas la sémantique qui détermine la pragmatique ${ }^{8}$.

2 En ce sens, Travis ne s'oppose pas frontalement à Frege, qui avait précisément distingué le sens et la vérité - cette dernière ne concernant pour sa part que les assertions en tant 
qu'elles expriment des jugements, et n'étant donc pas nécessaire pour identifier le sens d'autres types d'énoncés. Travis ré-utilise plutôt certaines analyses de Frege $^{9}$, qui accordent d'ailleurs une première place au contexte (« phrastique » et temporel ${ }^{10}$ ), pour les associer à celles d'Austin (lui-même fin lecteur et traducteur de Frege) afin de critiquer une certaine forme de conception de la représentation, qui vise souvent, en philosophie du langage, à penser la vérité d'une manière extrêmement robuste, et qui donne lieu à l'idée que c'est seulement en exprimant ou en portant des représentations singulières et totalement déterminées que le langage peut parler (correctement) du monde et donc avoir un sens.

3 Cette idée, qu'on peut retrouver tant chez le Wittgenstein du Tracatus que chez D. Davidson, on la formulera temporairement de la façon suivante, en reprenant la synthèse qu'en donne Travis: une représentation, qu'elle soit d'ordre mental (une idée, par exemple) ou linguistique (une phrase, par exemple), est une façon de représenter le monde (ou les choses). Une représentation est ainsi la représentation qu'elle est - elle a une identité - en raison de sa forme représentationnelle (c'est selon une certaine structure qu'elle représente le monde et cette structure n'est pas elle-même d'ordre linguistique ou physique). Par définition, la forme qu'elle détient est la sienne propre: aucune autre représentation ne peut l'avoir sans être cette représentation même ; représenter de cette manière, c'est nécessairement représenter comme elle le fait et c'est donc nécessairement être la même chose qu'elle. Par ailleurs, dans sa relation au monde, une représentation s'identifie par la façon dont elle représente l'état du monde (comment il est). L'état dans lequel il se trouve selon elle est ainsi fixé de manière déterminée par une représentation donnée. Elle le représente d'une certaine façon. De la même façon, une représentation est la représentation qu'elle est en fonction de ce qu'elle représente être le cas. Si elle représente autre chose, elle n'est plus la même représentation.

Dès lors, dans ce cadre, la question de la représentation a à voir avec celle de la vérité : représenter - et signifier, si signifier c'est représenter -, c'est représenter quelque chose et c'est représenter quelque chose d'une certaine façon, comme étant tel ou tel (c'est par exemple, attribuer une propriété à un sujet) ; la question étant alors : est-ce le cas ? La représentation représente-t-elle correctement? Si une représentation est telle qu'on vient de la définir, l'idée est qu'on peut déterminer de manière immédiate, ou " directe ", quand une représentation est correcte (ou « vraie ») - c'est-à-dire ses conditions de ses vérité $^{11}$. De telle sorte que la « face » représentationnelle de la représentation exige une et une seule « face » mondaine, fixée par ces conditions de vérité. Un seul état du monde est possible pour une représentation donnée, ayant l'identité qu'elle a.

5 Cet appel à la représentation, ainsi formée et que Travis appelle une " ombre », permet selon ses défenseurs de penser le rapport du langage au monde, puisqu'il semble que cette idée permet de comprendre immédiatement quand un énoncé est vrai, en déterminant de quoi il parle. Ladite représentation est alors identifiée à une "proposition» (au sens analytique d'un contenu de signification indépendant du temps et du lieu d'énonciation), via l'idée que la signification véritable (ou désambiguïsée) d'une phrase permet de déterminer uniment quand elle est vraie (ou qu'elle s'explicite en termes de conditions de vérité claires et précises). On pose ainsi l'hypothèse de propositions qui expriment de manière absolue le contenu d'un énoncé. Le représentationnalisme se transforme alors en platonisme, en ce qu'il considère que la représentation-proposition est censée déterminer quand elle serait vraie de manière 
absolue (sans qu'importe la façon dont le langage est engagé, ou en relation, avec le monde) ${ }^{12}$.

6 Or, Travis entend montrer qu'il n'est nul besoin de considérer qu'il existe des propositions de ce type pour comprendre comment l'on parle du monde et former des significations. Au moyen d'une argumentation qu'on appellera contextualiste ${ }^{13}$, en raison du recours constant à un phénomène qu'il appelle la « sensibilité à l'occasion » (occasionsensitivity), il va montrer, dans une perspective austinienne, que l'usage ordinaire des mots relèvent de pratiques, nécessairement contextuelles ou situées, qui suffisent à expliquer comment l'homme et le langage entrent en relation avec le monde.

7 En repartant de la conception frégéenne de la proposition, qui faisait elle-même place à l'intervention (minimale) d'une forme de pragmatique, nous entendons d'abord retracer rapidement comment s'est formée l'idée que la «représentation » est le porteur naturel de sens d'un énoncé en tant qu'elle permet d'en dégager les conditions dans lesquelles il est vrai, indexant ainsi la conception du sens à une détermination de la vérité associée à l'énonciation de cette proposition. Nous examinerons ensuite les critiques adressées par Travis à cette forme de pensée, telle qu'on peut par exemple la retrouver chez Davidson, avant de montrer que son contextualisme radical ne consiste pas à nier la dimension du sens, ni à refuser son rôle pour déterminer la vérité, mais plutôt à redonner un rôle important à nos pratiques (linguistiques, mais pas seulement) dans la détermination objective du contenu de ce qui est dit et son évaluation ${ }^{14}$.

\section{De la conception frégéenne de la signification et de la vérité à la conception vériconditionnelle de la signification : genèse de l'idée de "proposition »}

8 Pour comprendre le rôle que peuvent jouer les représentations dans la détermination de la signification, il peut être utile d'en retracer rapidement une sorte d'histoire conceptuelle. On la fait généralement remonter à Frege, qui, dans deux articles décisifs, a donné une définition de la signification qui permet, dans certaines conditions, de déterminer la vérité (et de comprendre ce qu'elle est).

i/ Précisons d'emblée que l'enjeu pour Frege était d'établir une sorte de langage idéal une idéographie - permettant d'établir les lois de l'être-vrai, c'est-à-dire les relations inférentielles permettant de sauvegarder la vérité ${ }^{15}$. Il concentre donc son analyse sur les énoncés qui visent la vérité, les "énoncés de la science " qui, d'un point de vue grammatical, forment autant d'assertions (ce sont des énoncés dont la forme grammaticale est généralement analysée comme l'attribution d'un prédicat à un sujet). Remarquons cependant que Frege ne réduit pas l'ensemble des énoncés à des assertions, mais seulement qu'il concentre l'analyse sur elles.

10 Une première étape (importante) de la réflexion de Frege est de distinguer (au moins) deux dimensions différentes de la signification des énoncés : la dimension du sens (Sinn) et celle de la référence (Bedeutung) ${ }^{16}$. La référence correspond à ce qui est désigné - par exemple, l'étoile réelle désignée par l'expression «L'étoile du Berger». Le sens est la dimension cognitive dans laquelle «le mode de l'être donné est contenu ${ }^{17}$; ou encore c'est « le mode de donation », c'est-à-dire la façon dont une expression désigne quelque chose ou y réfère. L'introduction de cette dimension est nécessaire, selon Frege, pour rendre compte du fait que l'on peut désigner la même chose par deux expressions 
différentes qui n'ont pas nécessairement la même signification. Par exemple, l'expression « l'étoile du nord » réfère au même objet (la même étoile) que l'expression «l'étoile du soir ", mais de façon différente. Cela s'explique parce qu'elles ont deux sens différents, qui donnent le même objet de deux façons différentes, l'une en le qualifiant d'étoile du matin et l'autre en le qualifiant d'étoile du soir. On comprend bien la différence si l'on remarque qu'une personne qui saurait ce qu'est l'étoile du matin sans savoir ce qu'est l'étoile du soir référerait, au moyen de l'expression «l'étoile du matin", à l'étoile désignée par "l'étoile du soir " sans accepter que cette dernière expression le fasse, puisqu'elle n'en comprendrait pas le sens. On comprend ainsi comment les noms propres, ou les expressions renvoyant à un objet, ont une double dimension signifiante : un sens et une référence.

Frege va généraliser ce point et considérer qu'on trouve dans ce modèle une façon de comprendre comment deux phrases différentes peuvent exprimer la même chose (par exemple, «le ciel est bleu » et "the sky is blue »; ou «François Mitterrand a été élu Président en 1981 » et « Le candidat socialiste a gagné les élections en 1981 »). De manière identique à l'exemple précédent, deux phrases peuvent être différentes quant à leurs éléments linguistiques et pourtant avoir le même contenu. Il faut donc là encore identifier autre chose que leurs seuls éléments linguistiques pour identifier ce qui est dit (le «contenu»). Frege va considérer qu'« une phrase assertive contient une pensée » [ Gedanke], laquelle correspond à ce qui est pensé en disant cette phrase, c'est-à-dire au contenu objectif de cette phrase (au sens où celui-ci n'est pas réductible à des représentations singulières, propres à une personne donnée, mais est au contraire partageable par n'importe quel penseur $\left.{ }^{18}\right)$. Sens et pensée finissent ainsi par être identifiés : une pensée est le sens d'une assertion qui l'exprime, sous une forme ou une autre. Ce faisant, une assertion exprime une " proposition", au sens d'une unité de sens qui, dès lors que sont fixés (au moins) le lieu et le temps qui s'y rapportent, est indéfiniment stabilisée. Ainsi, la pensée correspond à la proposition intemporelle exprimée par un énoncé et peut, de fait, être partageable par quiconque : quiconque la pense pense cette pensée, quelle que soit la façon dont il s'en saisit ou l'exprime individuellement. De la même façon, quiconque énonce une phrase exprimant cette proposition, une fois complétés les éventuels éléments indexicaux (lieu, temps, etc.) qu'elle comprend, signifie ce que cette proposition signifie et peut donc se faire comprendre par quiconque en saisit le sens, peu importe les particularités linguistiques de la phrase qui l'exprime.

Quelle est alors la référence d'un tel énoncé qui a du sens ? Précisons immédiatement que tout énoncé doué de sens n'a pas nécessairement de référence ${ }^{19}$. Seuls certains types d'énoncés, prononcés dans certaines conditions, ou dans certains contextes qui permettent de déterminer complètement et précisément la pensée exprimée, ont en une. Parmi eux, les assertions. Les assertions correspondent à l'exercice d'un jugement - et, à ce titre, expriment une pensée. Mais, au moyen de cet acte de jugement, elles ajoutent quelque chose à la pensée exprimée. Comme le dit Frege : « l'acte de juger peut être conçu comme la progression d'une pensée à sa valeur de vérité20 $»$.

On peut en effet exprimer une pensée sous la forme d'une prédication, de l'attribution d'une propriété à un sujet (exemple : «L'herbe est verte »). C'est véritablement l'acte de jugement qui rend compte de l'acte de prédication. Or, cela n'ajoute rien au sens de la phrase - car rien n'est ajouté au sens de la phrase du fait qu'elle est vraie. Bien plutôt, cet acte de jugement, tel qu'en rend compte une assertion, la dote d'une référence : sa valeur 
de vérité21 ${ }^{2}$ Cela conduit Frege à considérer que la référence d'une pensée est, non pas l'état de choses qui rendrait cette pensée vraie, mais la valeur de vérité du jugement porté, soit la vérité, soit la fausseté. En effet, ce qui est en jeu dans la référence d'une assertion, c'est un jugement, donc une relation entre l'énoncé et le réel - relation dont témoigne précisément l'assertion en tant qu'acte : l'assertion porte déjà en tant que telle l'acte de tenir pour vrai l'énoncé considéré et réfère donc à une valeur de vérité.

Mais, on le comprend, si Frege associe bien le sens d'une assertion avec une proposition déterminé et invariable, il n'associe pas étroitement le sens d'un énoncé à sa vérité, même si les énoncés assertifs ont pour ambition de référer à une valeur de vérité, c'est-à-dire de dire le vrai. Tous les énoncés n'ont en effet pas cette ambition et, même en ce qui concerne les assertions, celles-ci peuvent avoir un sens, c'est-à-dire exprimer une "proposition", sans que soit encore déterminée la valeur de vérité à laquelle elles réfèrent - puisque c'est précisément le sens des énoncés assertifs qui permet de saisir leur référence, en tant qu'ils expriment un jugement qui vient opérer la médiation entre le sens et la référence qu'est la valeur de vérité. Autrement dit, un élément nonsémantique - l'acte de juger, qu'on peut identifier à une pratique - doit intervenir pour mettre en relation un énoncé doué de sens et exprimant à ce titre une proposition avec une valeur de vérité qui sera la référence du jugement effectué, lequel est éventuellement exprimé par l'assertion de cette proposition.

Par la suite, cependant, la conception frégéenne va être reprise et légèrement modifiée pour donner lieu à une formulation standard qui va associer beaucoup plus étroitement la proposition exprimée et sa véritée ${ }^{2}$, en refoulant la première dimension pragmatique introduite par Frege au moyen de l'acte de jugement.

ii/ Ainsi, quelques années plus tard, L. Wittgenstein a pu exprimer de manière très directe, dans le Tractatus Logico-philosophicus, une sorte de formulation canonique du sens associant étroitement " proposition » et " conditions de vérité » :

4.021 - La proposition est une image de la réalité. Car je connais par elle la situation qu'elle présente, quand je comprends la proposition. [...]

4.022 - La proposition montre son sens.

La proposition montre ce qu'il en est des états de choses quand elle est vraie. Et elle dit qu'il en est ainsi.

4.023 - La réalité doit être fixée par oui ou par non grâce à la proposition.

Il faut pour cela qu'elle soit complètement décrite par la proposition.

La proposition est la description d'un état de choses. [...]

4.024 - Comprendre une proposition, c'est savoir ce qui a lieu quand elle est vraie. (On peut donc la comprendre sans savoir si elle est vraie). On peut donc la comprendre quand on comprend ses constituants [...]

4.06 - La proposition ne peut être vraie ou fausse que dans la mesure où elle est une image de la réalitéé

Ici, le sens, ou la signification, d'un énoncé est clairement rapporté à une " proposition » qui est elle-même indexée sur le réel via des conditions de vérité déterminées. La proposition est précisément ce qui, dans le langage, permet de déterminer tout à la fois le réel dont elle parle et si elle parle correctement du réel, en fonction des conditions de vérité qui lui sont corrélées et qui déterminent étroitement le réel (ou la "référence ») auquel elle renvoie - comme si le langage pouvait de lui-même fixer le critère de ce qui le vérifie ou de ce qui lui correspond. On retrouvera cette conception, qui associe étroitement le sens et la référence au moyen de la proposition, chez de nombreux auteurs ${ }^{24}$ et notamment, de manière exemplaire, chez Davidson. Selon lui, par exemple, " la vérité d'un énoncé ne dépend que de deux choses : ce que signifient les mots tels qu'on les 
dit et la manière dont le monde est organisé. Elle n'est pas, en prime, relative à un schème conceptuel, une façon de voir les choses ou une perspective ${ }^{25}$. "

L'idée est que la signification des mots suffit à elle seule à déterminer quelle est la bonne application d'un énoncé composé de ces mots, en ce qu'elle fixe ses conditions de vérité. Plus précisément, $c^{\prime}$ est la signification de chacun des mots qui composent une expression donnée qui, en raison de l'hypothèse de compositionnalité du sens, détermine la façon dont un énoncé signifie quelque chose, c'est-à-dire représente quelque chose comme étant le cas. Un énoncé comme « Le chat est sur le tapis » signifie en effet que le chat est sur le tapis et, à ce titre, représente le fait, pour un chat, d'être sur un tapis. Or, suivant cette idée, on peut le modéliser par certaines conditions de vérité particulière: l'existence d'un chat, d'un tapis et la présence du chat sur le tapis. L'énoncé est alors dit avoir une forme représentationnelle, modélisée par ces conditions de vérités. Celles-ci spécifient une façon, pour l'énoncé, de représenter quelque chose, c'est-à-dire de dire que quelque est le cas, ou est tel ou tel (d'attribuer une propriété à un sujet, par exemple). On comprend ainsi que la structure représentationnelle d'un énoncé dépend de la structure de ses conditions de vérité, qui elles-mêmes dérivent de sa signification. C'est donc, pourrait-on dire, en dernière (ou en première) instance, la signification qui détermine la structure représentationnelle d'une phrase ${ }^{26}$.

iii/ Or, cette première idée conduit à deux autres idées, qui mènent à ce qu'on peut appeler avec Travis une forme de "platonisme linguistique $»^{27}: 1$ ) l'idée qu'on peut décompter de manière précise toutes les significations à rencontrer dans le monde, c'està-dire l'idée qu'il existe un stock de significations ayant une identité propre et qui sont étanches les unes aux autres ; 2) l'idée, déjà présente chez Frege, que le porteur de ces significations, et donc des structures représentationnelles associées, n'est pas l'énoncé lui-même (ni même la phrase utilisée) mais une forme plus abstraite, que l'énoncé exprime: la "proposition». Ces deux idées associées conduisent à l'idée que pour comprendre ce qu'un énoncé veut dire, il faut saisir la "proposition " qu'il exprime, parmi le stock de propositions possibles qu'il peut exprimer, étant donnés les mots qui le forment.

En effet, si on regarde l'usage ordinaire du langage naturel, on se rend compte que les énoncés qui le composent ne sont pas eux-mêmes dotés des structures représentationnelles qui les associeraient strictement avec un état de choses donné, ou avec des conditions de vérité déterminées. Un même énoncé est souvent utilisé pour dire des choses différentes et donc pour renvoyer à des états du monde différents. Il semble ainsi pouvoir posséder plusieurs structures représentationnelles. Une idée largement répandue consiste alors à considérer que les énoncés du langage ordinaire souffrent d'ambiguïté, c'est-à-dire qu'ils expriment, selon les circonstances, différentes significations. Pour rendre compte de ce phénomène, on n'accorde pas de rôle aux circonstances, mais on considère que les mots qui composent l'énoncé sont susceptibles d'exprimer différentes choses, selon un certain nombre de paramètres, eux-mêmes déterminés par la signification des mots. Deux modèles sont disponibles pour expliquer ce phénomène : le modèle de l'indexicalité, inspiré de Frege et retravaillé par D. Kaplan ${ }^{28}$, et le modèle de l'ambiguïté. Le modèle de l'indexicalité considère que certains termes (indexicaux, tels que « je », « maintenant », etc.) gagnent un plein contenu en fonction de leur usage (ces termes sont pensés comme des variables qui gagnent un contenu selon une certaine fonction). Ce contenu s'obtient en fonction de certains paramètres, déterminés par la signification même : ainsi, le mot « je » réfère à la personne qui l'utilise, 
etc. Ces paramètres donnent à toute valeur que peut prendre le terme indexical un et un seul contenu précis qui serait exprimé par la phrase le contenant lors d'un usage donné.

Le modèle de l'ambiguïté considère pour sa part que la signification d'un terme ou d'une phrase ne peut pas déterminer à elle seule ce qui est exprimé en une occasion donnée (d'où l'ambiguïté de certains énoncés), mais qu'elle fournit néanmoins un ensemble déterminé de contenus distincts, dotés d'une identité signifiante pleinement déterminée, qui sont susceptibles d'être exprimés en cette occasion. Ce qu'exprime la phrase utilisée en une occasion est donc à choisir parmi ce stock défini de contenus déterminés disponibles, qui se situent en-deçà ou au-delà de ce qui est dit et qui reste ambigu.

Dans les deux cas, on va donc considérer qu'à un niveau situé au-delà de la structure purement linguistique, se trouve une autre structure pleinement déterminée qui explicite ce qui est vraiment dit. Cette structure représentationnelle est précisément ce qu'on appelle une " proposition »; laquelle, selon Travis, n'est qu'une « ombre " posée derrière le langage lui-même pour rendre compte de son fonctionnement ${ }^{29}$. L'idée est en effet que cette proposition, en raison de l'identité réprésentationnelle définie qu'elle détient (qui fait qu'elle est la proposition qu'elle est, et non pas une autre; qui fait qu'on ne peut pas la confondre avec une autre proposition, qui peut, à l'occasion être exprimée par la même phrase), détermine totalement, c'est-à-dire de manière non-ambiguë, quand elle serait vraie, c'est-à-dire l'état du monde dont elle parle ${ }^{30}$.

Il suffirait donc de saisir, ou d'exprimer, une proposition au moyen du langage naturel pour pouvoir parler du monde de manière définie. Comme le dit Travis :

Considérons qu'une assertion donnée, "Les cochons grognent", possède une formepour-l'occasion (nonce-form) spécifiable en spécifiant une chose particulière qu'on pourrait comprendre comme susceptible d'être un grogneur. Nous pourrions alors introduire un nouveau prédicat dans le langage, disons «- plogne » et le doter de cette forme quand il signifie ce qu'il signifie. Nous pourrions alors simplement dire " plogner, c'est être un grogneur en ce sens ». Si cette forme-pour-l'occasion est une ombre, alors "plogner ", en signifiant ce qu'il signifie, se comportera comme un prédicat doit se comporter s'il répond à la conception ombresque (et donc la conception davidsonienne) de l'engagement avec le monde. Elle n'admettra pas deux compréhensions rivales, qui ne seraient ni l'une ni l'autre incorrectes. On ne se fera jamais deux idées différentes quant à ce qui est ou non décrit par " plogne » 31 .

On obtient alors bien une détermination précise et unique de ce qui est dit en posant des propositions fonctionnant comme des ombres du langage. Reste que le problème d'une telle conception, c'est, comme ajoute immédiatement Travis, qu'elle « souligne seulement combien " - plogne" serait peu ressemblant avec n'importe quel prédicat familier du français $»^{32}$

\section{Arguments contextualistes et anti- représentationnalistes}

Cette absence de familiarité avec la façon dont fonctionnent les prédicats du langage naturel (et, plus encore, ordinaire) est ce sur quoi Travis va s'appuyer pour défendre une conception contextualiste de la signification et une conception pragmatique de la vérité. Celles-ci reposent sur une critique, elle-même contextuelle, de la position précédemment explicitée, qu'on peut résumer en deux points: 1) une critique des présupposés qui mènent à l'idée qu'il existe des formes représentationnelles (des " propositions ») du type 
exposé ci-dessus ; 2) la démonstration du fait que la conception précédente ne garantit pas, et ne peut pas garantir, la solution qu'elle entend fournir. Il s'ensuivra comme conséquence l'inutilité du recours à des représentations-ombres pour penser le rapport du langage et du monde.

i/ L'argumentation centrale de Travis consiste à montrer que les constructions théoriques précédentes reposent toutes sur l'idée que toute distinction repérée à une occasion donnée est une distinction pérenne, ou essentielle. L'idée qui sous-tend la recherche d'une proposition qui identifierait de façon précise ce qui est dit (c'est-à-dire le contenu exprimé), est l'idée que, pour comprendre (ou saisir, ou encore appréhender) le contenu d'un énoncé, il convient d'identifier une structure précise, totalement déterminée et déterminante, se rapportant de manière déterminée au monde. Autrement dit, pour tout état du monde dont on parle, on doit pouvoir trouver une et une seule structure correspondante, cette correspondance étant pensée de manière strictement biunivoque: à un élément d'un ensemble correspond un autre élément de l'autre ensemble. Comme le dit Travis :

L'idée qui anime ici le mécanisme de pensée est qu'une différence dans la façon de représenter les choses qui correspond une fois à ce qu'il y ait deux choses différentes, y correspond toujours. Indépendamment des circonstances, il n'y aurait qu'une seule bonne façon de classifier les cas considérés comme des cas où l'on pense, ou dit, la même chose ou différentes choses, ou des cas où l'on dit précisément ce que, ou autre chose que ce que quelqu'un pense, ou des cas où l'on pense à ce que quelqu'un a dit. [...] Si une assertion ne dit ce qu'elle dit qu'à rentrer en relation avec un item de ce type, alors, en toute occasion, c'est toujours la même chose qu'il y a à dire, ou qu'il faut considérer qu'une assertion dit. Dire ce qu'elle dit, pour une assertion, cela consiste toujours pour ce qu'elle a dit à faire contraste, des façons exactes, et avec la même variété de choses qui peut être dite. Il n'y a donc qu'un ensemble unique de traits d'une assertion qui, indépendamment de l'occasion, individualise précisément cet item qui est celui exprimé. ${ }^{33}$

Ce serait donc parce qu'on recherche des significations absolument individualisées que l'on en vient à poser des représentations fonctionnant comme des ombres, c'est-à-dire absolument dépourvues d'ambiguïté. Or, rien n'oblige à considérer que tout contraste noté entre deux façons de comprendre (ou de dire) les choses est un contraste absolu, pérenne ou essentiel. Travis s'attache ainsi à montrer que toute distinction posée peut être comprise comme étant "occasion-sensitive ", sensible à l'occasion, et qu'il n'y a donc pas besoin de poser la différence comme différence essentielle (par exemple, chez Frege, deux Sinne, deux choses dites ${ }^{34}$ ), mais qu'il suffit d'en rendre compte en fonction du contexte. Supposons en effet, pour reprendre un exemple de Travis, qu'on distingue entre le fait qu'un objet soit bleu et le fait que ce même objet soit peint en bleu. En une certaine occasion de décrire le visage d'un sorcier indien, il peut arriver que l'on fasse cette distinction («non, il ne s'étouffe pas, il s'est juste peint le visage »). Dès lors, un objet tombe soit exclusivement sous la catégorie des choses bleues (il peut être dit «bleu»), soit exclusivement sous la catégorie des choses peintes en bleu (il peut dit «peint en bleu»). Et si l'objet est bleu, il ne peut pas être dit à raison "peint en bleu», et inversement : un objet peint en bleu ne peut pas, dans ce cas, être considéré comme bleu. Or, ce que montre Travis, c'est que ce cas ne fixe pas toutes les possibilités d'opérer, ou non, la distinction entre les choses bleues et les choses peintes en bleu. Il y a en effet des cas, en d'autres occasions, où la distinction entre le fait d'être bleu et le fait d'être peint en bleu ne s'applique pas. Si, par exemple, je cherche à savoir de quelle couleur est ta voiture et que tu me dis " Elle est bleue ", je considérerais que cette assertion est vraie si 
ta voiture est peinte en bleue. Le fait d'être bleu admet ainsi en cette occasion une compréhension qui permet qu'on l'applique aux choses peintes en bleu.

Il est de fait erroné de penser que, dès lors qu'on note une distinction faite entre deux façons de comprendre un même énoncé, il faut essentialiser cette distinction pour en faire une disjonction nécessaire entre deux choses à dire (et poser deux " propositions » correspondantes). Ce n'est pas parce qu'un énoncé, ou un concept, admet en une occasion deux compréhensions différentes - et mutuellement exclusives - qu'il l'admet toujours. La distinction à faire est relative à l'occasion de la faire et peut très bien ne pas être faite dans d'autres circonstances. Autrement dit, l'idée qu'il faille faire correspondre des " propositions » différentes à chaque contenu exprimé par un énoncé ne tient pas compte du fait que chaque contenu dépend de la façon dont on comprend l'énoncé en une occasion donnée et que cette façon peut varier. Il est donc abusif de vouloir poser des propositions singulières correspondant à chaque compréhension, alors que le contenu qu'elles sont censées identifier dépend essentiellement des circonstances de l'énonciation.

ii/ Maintenant, à supposer que les conceptions qui font des propositions des ombres de nos pratiques soient justifiées à le faire, l'hypothèse de telles ombres ne règle de toute façon pas le problème qu'elles sont censées résoudre. En effet, l'idée est de trouver des items qui soient dotés de structures représentationnelles telles qu'elles ne laissent aucune place à l'ambiguïté, de telle sorte qu'on puisse toujours identifier immédiatement de quoi parle une proposition (à travers ses conditions de vérité). Cela suppose donc qu'on ne puisse jamais comprendre ou saisir une proposition autrement qu'elle ne « demande » à être saisie. Or, Travis va montrer que c'est précisément là une chose que le recours à des représentations-ombres, ou des " propositions", ne peut pas garantir.

Une façon de le faire consiste à montrer, dans un mouvement lui-même frégéen, que les structures envisagées ne permettent pas de dire quand elles seraient vraies. (Il faut montrer que n'importe quelle structure de ce type que nos mots peuvent avoir ne marche pas.) Pour montrer qu'une structure de ce type ne marche pas, il suffit de montrer que, pour toute phrase signifiante, susceptible d'être vraie, et donc ayant censément une structure représentationnelle à exprimer lui permettant d'être vraie, il est possible que cette phrase soit vraie à propos d'une chose, en une occasion de la dire, et soit fausse à propos de la même chose (ou du même état de fait) en une autre occasion de la dire. Autrement dit, il s'agit de montrer que, pour toute assertion, le prédicat ou le concept qu'on veut appliquer admet plusieurs compréhensions, non nécessairement compatibles.

Or, il est facile de le faire avec un cas simple et exemplaire. Prenons la phrase « Le chat est sur le tapis ». Supposons qu'elle serve à exprimer la proposition que le chat est sur le tapis, dotée d'une certaine structure représentationnelle donnée, identifiée par certaines conditions de vérité. En l'occurrence : "le chat est sur le tapis » est vraie ssi le chat est sur le tapis. Toute la question est de savoir si la compréhension de cette proposition est absolument déterminée par le fait de poser cette condition de vérité. Il est facile de voir que ce n'est pas le cas. Il est en effet possible de comprendre cette proposition de deux façons différentes (au moins) et mutuellement exclusives. En une occasion donnée (par exemple, je réponds à ma petite s?ur qui cherche sa peluche), je peux utiliser cette phrase, exprimant cette proposition, pour dire que son chat en peluche est sur le tapis. En une autre occasion donnée (nous voulons nous assurer que le chat n'est pas dans la machine à laver), je peux utiliser cette même phrase, exprimant la même proposition, pour dire que le chat se trouve sur le tapis. Or, je ne peux pas vouloir dire la même chose 
dans les deux cas : dans le deuxième cas, il n'est pas vrai que le chat est sur le tapis si c'est la peluche de ma sœur qui s'y trouve. Pour autant, les deux usages sont parfaitement légitimes - précisément parce que l'hypothèse d'une "proposition» ne suffit pas à bloquer les compréhensions admissibles qu'elle peut recevoir en contexte.

Or, Travis soutient qu'aucune position d'un niveau d'abstraction supplémentaire dans la structure représentationnelle ne suffira à empêcher de phénomène d'interprétation contextuelle (lié ici aux intérêts en jeu dans la description). Il suffit pour le montrer de montrer qu'un mot donné, ou qu'un prédicat donné, ayant des conditions de vérité spécifiées, peut toujours admettre plusieurs cas contradictoires. Par conséquent, les "propositions », dotées de la structure représentationnelle considérée auparavant, ne parviennent pas à identifier un état donné des choses auquel elles s'appliquent et ne permettent pas à elles seules de déterminer quand elles seraient vraies. Le fait de poser de telles structures représentationnelles apparaît donc inutile.

Ainsi, dans certains cas, deux personnes peuvent tout à fait considérer différemment une même phrase, même si la proposition qu'elle exprime est précisément identifiée, tout en étant justifiées à le faire dès lors qu'elles explicitent la façon dont elles comprennent le concept utilisé. On a alors affaire à l'application d'une proposition et donc à l'intervention du domaine pragmatique dans la détermination de ce qui est dit en une occasion donnée. On en vient donc à admettre que c'est peut-être finalement la compréhension qu'on a de certains termes en une certaine occasion qui permet de spécifier quand ces termes seraient vrais.

34 Car montrer que l'hypothèse des propositions ne permet jamais de désambiguïser absolument la compréhension qu'admet un énoncé, cela ne suffit pas à montrer que ce n'est pas selon ce schéma que fonctionne le langage. Il convient encore de montrer l'inutilité de propositions de ce type et autres «ombres» en expliquant comment le contexte suffit à faire le travail de désambigüisation et d'identification d'un contenu précis, qui se rapporte au monde d'une façon déterminée.

\section{Le rôle pragmatique du contexte dans la détermination de la signification et de la vérité}

i/ Pour Travis, malgré ces critiques, on identifie bien quelque chose comme une " proposition » (à savoir le sens d'une phrase, ou ce qu'elle dit) en fonction de ce qui est le cas selon elle; mais c'est là une chose qui dépend de la compréhension qu'on a d'un énoncé à chaque occasion singulière de le dire. Autrement dit, cette identification repose plutôt sur des compréhensions, relatives au contexte et à l'occasion ${ }^{35}$.

Il ne s'agit pas là de ré-introduire, un degré plus haut ou un cran derrière, une structure représentationnelle (enfin) fixe - la proposition-comprise-en-un-certain-sens. Il ne s'agit pas non plus de dire qu'il y a dans chaque "proposition» identifiée une ambiguïté à désambiguïser, comme s'il y avait différentes structures clairement identifiables derrière une structure floue, et que pour chaque structure identifiée on pouvait déterminer précisément quand elle serait vraie. Bref, il ne s'agit pas de déterminer un ensemble d'options admissibles au moyen du phénomène contextuel de compréhensions multiples admises, comme s'il s'agissait encore des résultats calculables d'une fonction selon la valeur que prendraient de nouvelles variables. Il s'agit simplement de comprendre qu'en une occasion (c'est-à-dire en une occasion d'usage), on peut bien identifier un énoncé en 
fonction de ce qu'il dit à propos du monde - ou, si l'on y tient, de ce qu'il « représente » (car une phrase peut bien être un outil représentationnel). Comment alors spécifier ce « contenu»?

Il faut comprendre que le contexte (tout à la fois circonstancié et historique) relatif à l'occasion d'utiliser chaque énoncé peut suffire à fournir des raisons de comprendre telle ou telle chose, en fonction de l'énoncé utilisé, plutôt que telle autre. Un énoncé donné, par exemple, «Les feuilles sont vertes ", utilisé pour faire une assertion, possède en effet bien une signification (un contenu sémantique ou représentationnel : il parle des feuilles et de la couleur verte qui leur est attribuée), qui est construite à partir du sens littéral des mots qui le composent. Ce contenu sémantique "constant " peut être fixé de manière conventionnelle, ou de manière naturelle, peu importe ici. Ce qui importe est que sa contribution ne suffit pas à déterminer ni l'ensemble de ce qu'il dit, ni quand l'énoncé serait vrai (ou utilisé correctement, selon ce qu'on veut faire avec lui). Comme on l'a vu précédemment, il existe toujours de multiples compréhensions admissibles d'un contenu sémantique selon le contexte d'énonciation. Travis précise: "ce qu'un énoncé dit lors d'une énonciation, de certaines feuilles, n'est pas seulement déterminé par ce qu'il signifie ou par ce que signifient les éléments qui le composent $»^{36}$ : il faut en effet distinguer entre ce qui est signifié (donné par le lexique) et ce qui est dit par un énoncé. Mais «ce qui est dit" n'est pas à rechercher à un niveau antérieur ou supérieur à l'énoncé, comme si cela attendait d'être découvert. Cela est construit par le contexte d'énonciation qui détermine des façons de comprendre l'énoncé, qui ne sont pas nécessairement anticipables (hors-contexte).

Ainsi annoncée, l'intervention du contexte pourrait faire sembler courir un risque à la possibilité de la détermination du contenu et empêcher toute recherche sur la sémantique du langage. Selon Travis, l'intervention du contexte est en fait ce qui vient déterminer (en plus de leur sémantique) le contenu des mots utilisés. Car le contexte vient spécifier les buts, les raisons pour lesquels tel énoncé est utilisé, en même temps que les traits contextuels pertinents (relatifs par exemple à l'histoire de la discussion menée, ou de la famille dans laquelle se tient cette discussion, etc.). Ainsi, si dans l'absolu on peut comprendre de multiples choses différentes et incompatibles par l'énoncé selon lequel l'eau du lac est bleue, en contexte, certaines compréhensions sont plus admissibles que d'autres, sont plus motivées. On peut même considérer que certaines s'imposent en raison du contexte : si on me dit que l'eau du lac est bleue au cours d'un examen visant à analyser si de la peinture bleue s'est déversée dans le lac, je ne comprendrais pas l'énoncé comme voulant dire que l'eau du lac apparaît bleutée au soleil. Cela ne serait pas raisonnable ou pas pertinent, au regard du contexte d'énonciation, c'est-à-dire eu égard aux autres informations que j'ai, relativement à la pratique dans laquelle je suis engagée, étant donnée les objectifs pratiques qui sont les miens à ce moment (Puis-je me colorer en bleu un feuille de papier ?), etc.. Inversement, si on me dit que l'eau du lac est bleue alors qu'on prévoit d'aller s'y baigner, je ne comprendrais pas cet énoncé comme voulant dire que, si je bois de l'eau du lac, celle-ci sera bleue. Il s'agit là de deux situations différentes où la même phrase est employée pour dire deux choses différentes. Et les contenus respectifs portés par chaque énonciation en chaque occasion sont en fait générés par les pratiques dans lesquelles les énonciations s'inscrivent, qui viennent d'une certaine façon «motiver» la façon dont il faut prendre les énoncés ${ }^{37}$. Ainsi, ce que fournit le contexte, ou la sensibilité à l'occasion, c'est une façon de comprendre ce qui est dit à partir du contenu sémantique, ou « représentationnel », des mots prononcés. 

beaucoup de choses différentes sont susceptibles de l'être et ce sont les pratiques des hommes qui parlent qui viennent spécifier comment il faut entendre ce qui est dit. Cela seul permet d'identifier ce dont on parle précisément ${ }^{38}$.

faudrait pourtant pas s'imaginer, en cédant à nouveau à la pulsion du platonisme, qu'on pourrait identifier l'ensemble des traits contextuels qui peuvent intervenir dans la détermination du contenu (des « compréhensions » qu'on en a), de manière par exemple à pouvoir en faire les variables, aussi multiples soient-elles, d'une fonction compliquée. Cela est impossible, car il n'existe pas de façon de prévoir comment le contexte, en une occasion donnée, vient spécifier comment il faut prendre ce qui est dit : autrement dit, il n'existe pas une liste donnée des compréhensions possibles admissibles dans une situation, selon une liste de traits pertinents à retenir, etc. ${ }^{39}$. La liste des situations n'existe pas plus que la liste exhaustive des traits à retenir, ni donc que celle des compréhensions admissibles. Il n'existe tout simplement pas de façon a priori, déterminée, dont on doit comprendre les mots en une occasion donnée (d'où le contextualisme radical de Travis) ${ }^{40}$. Cela dépend précisément du contexte et de la façon dont nous, humains, réagissons dans ce même contexte, sachant que ce même contexte peut admettre, en fonction des intérêts de chacun, des motifs, des histoires, etc. différentes postures quant aux mots utilisés et donc différents perspectives prises quant à la façon de les comprendre, toutes justifiées selon les occasions. Cela dépend donc « de la façon dont nous menons nos vies $»^{41}$, laquelle peut être plus moins fixée, normée, régulée, conventionnelle, etc. Autrement dit, Travis nous renvoie, dans un mouvement volontairement aristotélisant, à la raison, aux habitudes des êtres humains, etc., plutôt qu'à leur rationalité calculatrice ${ }^{42}$.

41 Reste pourtant qu'une telle conception du sens de nos mots, renvoyant sa détermination complète au contexte de leurs énonciations, semble immédiatement faire peser une menace de relativisme, ou du moins de désaccord, quant à leur vérité. Peut-on encore seulement dire la vérité au moyen du langage dans une telle conception, et si oui comment?

ii/ Sans aborder cette question de manière exhaustive ici $^{43}$, on peut dire que la vérité va devenir une notion elle-même contextualisée et pragmatisée. Travis entend reprendre le projet austinien consistant à briser le "fétiche fait/valeur" sans remettre en cause l'objectivité de la vérité : « la remise en cause du fétiche fait/valeur n'est pas la remise en cause des faits ", rappelle-t-il ${ }^{44}$. Il s'agit plutôt de comprendre que la question de la vérité va maintenant devoir se spécifier en fonction de trois facteurs, comme Frege le proposait déjà, plutôt que deux : la vérité va ainsi devenir une relation entre la signification des mots, l'état du monde et les circonstances d'énonciation. Car la solution ne peut plus consister à donner, a priori, les déterminations d'une compréhension permettant de dire si une compréhension donnée est correcte ou pas selon l'état du monde envisagé précisément parce que seul le contexte d'usage peut le faire. Comme le dit Austin, tout énoncé n'est pas vrai ou faux, cela dépend à la fois de ce que nous en faisons et de l'occasion de déterminer s'il l'est ${ }^{45}$.

43 Pour revenir à Travis, ce qui détermine la façon dont on va parler du monde, c'est précisément la façon dont, à chaque occasion, en contexte, nous appréhendons le monde, laquelle façon nous permet de choisir de le représenter de telle ou telle manière (dans notre langage, avec tels ou tels mots), selon les objectifs poursuivis. Interviennent également dans ce choix ce qu'on peut appeler notre « santé mentale» (sanity) et notre

Corela, HS-14 | 2013 
connaissance du monde, notre connaissance de la façon dont les gens vivent et réagissent. Celles-ci forment autant de raisons pour un locuteur de penser ou conclure telle ou telle chose, qui permettent d'identifier «tous les faits concernant ce qui, selon une compréhension, vaudrait comme le fait d'être comme ces mots le disaient $»^{46}$. En fait, cela dépend des «formes de vie » et de la façon dont celles-ci conditionnent la façon dont on va agir selon la façon dont on comprend les mots. On mesure ainsi la valeur d'une information en fonction de ce qu'on doit en attendre, étant donné nos objectifs, intérêts, etc., et selon la façon dont on utilise les mots (ce que Austin a appelé les différentes " forces illocutoires ${ }^{47}$.) Il existe tout un ensemble d'attentes qui conditionnent la façon dont, en une occasion, on va prendre tels mots (sans, là encore, qu'on puisse établir a priori une liste de ces attentes). Mais comment cela fonctionne-t-il en une occasion précise où je fais une assertion (et pas un autre acte de parole); comment être sûr que je dis correctement ce que je dis, que je dis vrai à propos du monde?

Supposons qu'en une occasion, je fasse une assertion et que j'entende par là dire la vérité. La vérité de mon énoncé va donc s'évaluer à propos de ce dont il parle en cette occasion précisément, car le contexte va venir spécifier mon énoncé de façon à le faire référer à cette réalité précise. Mais il va aussi permettre de comprendre comment j'entends précisément qualifier ce dont je parle, selon quels critères de finesse, de précision, etc. Supposons ainsi que je dise "L'eau du lac est bleue", en parlant du Lac Léman, en répondant à ma sœur qui s'apprête à aller y plonger et s'inquiète de la présence d'algues à cause des irritations qu'elles provoquent sur sa peau. Dis-je la vérité, mon énoncé (qui est ici une assertion) peut-il être qualifié de vrai ? Peut-on dire «L'eau du lac est bleue » est vrai? Dans ce contexte, au regard des pratiques (s'informer entre proches, dans une relation de complicité, nager, etc.) dans lesquelles nous sommes engagées (telles qu'elles sont définies dans notre milieu social, etc.), il est tout à fait possible de répondre positivement à cette question : je parle bien de la même eau et du même lac que ma s?ur (on peut s'en assurer, d'autres éléments en attestent, je viens de promener le chien le long de celui-ci, etc.); je la qualifie de bleue au sens où ma s?ur l'entend - au sens où sa couleur turquoise indique que la présence d'algue n'en altère pas le reflet bleuté au soleil - et je dis le vrai à ce qui est par là désigné : il est vrai que l'eau du lac est bleue s'il s'agit pour ma s?ur de savoir si elle peut aller y nager sans plonger dans un amas d'algues gluantes - du moins n'apparaissent-elles pas à la surface, ce qui est le seul critère selon lequel je puisse en juger à cette distance. Mais si ma sœur avait plutôt voulu connaître la couleur de l'eau du lac pour savoir si elle convenait pour repeindre la porte de la maison, alors je n'aurais pas dit vrai (même si je n'aurais peut-être pas dit faux ${ }^{48}$ ).

Cet exemple permet de comprendre deux choses à propos de l'énonciation de la vérité : premièrement, qu'à travers mon énoncé je m'engage vis-à-vis de la réalité considérée (je fais référence à cette réalité et à rien d'autre), car si on me demande des compte, je dois être capable d'en rendre pour cette réalité précise ; deuxièmement et en conséquence, qu'une véritable objectivité se dégage de ce processus d'affirmation de la vérité ; ou que la vérité, pour reprendre les termes de Travis, est une affaire « substantielle ». Dire en effet qu'une assertion est vraie, c'est encore prendre une responsabilité, puisque c'est prendre une responsabilité quant à ce qui est le cas et quant à la façon dont il est dit que c'est le cas. En ce sens, c'est bien en tant que nous sommes des êtres humains, qui nous inscrivons dans une temporalité et une localisation, ayant des intérêts multiples, que nous pouvons dire la vérité, aussi paradoxal ce résultat puisse-t-il paraître. 

qui consiste pour un jugement à être vrai, et du même coup la véritable responsabilité une distinction intelligible entre le fait d'avoir raison et le fait de simplement le sembler disparaît. C'est ainsi le fait de nous ôter ce rôle qui nous fait perdre en même temps le jugement. $»^{49} C^{\prime}$ est dire qu'il doit exister des critères qui permettent de distinguer ce qui est vrai et ce qui ne l'est pas, et qui permettent donc de juger objectivement. Reste que ces critères forment des standards de correction de la connaissance qui sont toujours paroissiaux, locaux, indexés à des pratiques; et c'est précisément parce qu'ils sont paroissiaux qu'ils nous permettent d'avoir une véritable connaissance objective. "Le paroissial, selon la conception de Wittgenstein [et d'Austin], est précisément ce qui permet à la responsabilité, à l'engagement, d'être ce qui revient aux mots, de telle sorte que, de façon correspondante, ils peuvent délivrer des engagements - des engagements à considérer les choses comme étant dans un état possible. Ce sont précisément eux qui permettent au monde de décider si les choses sont comme on a dit qu'elles étaient. Loin d'abolir l'objectivité, le paroissial en est ainsi précisément la source $»^{50}$.

\section{Conclusion : le contextualisme est un humanisme}

Dire la vérité n'est donc ni ce à quoi le langage est destiné en raison des structures propositionnelles qu'il exprime, ni un exploit particulier réalisé au gré des circonstances. Cela dépend simplement de ce qu'on veut faire avec l'énoncé, c'est-à-dire des « circonstances » de son usage, qui viennent ainsi spécifier la façon dont doivent êtres satisfaites les conditions données par la signification des mots utilisés, relativement à certains objectifs, à certaines attentes des humains engagés dans la situation - objectifs et attentes généralement corrélés aux usages connus des mots.

Autrement dit, il ne suffit pas de disposer de deux éléments - la signification d'une phrase d'un côté (qui représente le monde), l'état du monde de l'autre - pour déterminer si une phrase est vraie. Un troisième élément doit intervenir: les circonstances d'usage qui déterminent comment l'énoncé pourrait être satisfait (étant donnés les deux premiers éléments). Ce qui suppose, de la part des locuteurs, une certaine sensibilité aux circonstances, leur permettant d'établir comment la vérité pourrait à cette occasion être établie. De telle sorte que l'on comprend bien que la vérité n'est pas la propriété sémantique (ou représentationnelle) d'une phrase, mais plutôt la propriété (déjà repérée par Frege) d'évaluation pragmatique, par un locuteur situé, d'un énoncé proféré lors d'une occasion d'usage, déterminant lui-même le type d'attentes à remplir pour que sa vérité soit établie. De même, l'équivalence immédiate posée entre signification et conditions de vérité se trouve brisée, puisque la signification à elle seule ne permet plus d'établir ce qui rendrait vraie la phrase qui en est dotée. Bien plutôt, la vérité (d'un énoncé) ne peut être établie que par un locuteur donné évaluant l'usage d'une phrase dans un contexte précis, pour répondre à des attentes déterminées, en donnant telle ou telle description du monde qui l'environnait dans les circonstances où elle a été produite. Il s'agit là d'une production proprement humaine, mettant en jeu des facultés et des attentes typiquement humaines, liées à certaines activités mettant en jeu certains intérêts, dans lesquelles le langage est un outil pour l'atteindre sans y être nécessairement destiné.

Corela, HS-14 | 2013 


\section{BIBLIOGRAPHIE}

Al-Saleh, Ch. \& Laugier, S. (éd.) :J.L. Austin et la philosophie du langage ordinaire, Hildesheim : Olms, 2011.

Ambroise, B. : Les pouvoirs du langage : la contribution de J.L. Austin à une conception contextualiste des actes de parole, Thèse de doctorat de l'Université Paris X - Nanterre, 2005, 392 p.

Austin, J.L. : Philosophical Papers, edited by J.O. Urmson and G.J. Warnock, Oxford University Press, "Clarendon Press », Oxford, $3{ }^{\text {ème }}$ édition : 1979 ( 1 ère édition : 1961) ; trad. fr. partielle de L. Aubert et A.-L. Hacker, Ecrits philosophiques, Paris : Editions du Seuil, 1994.

Austin, J.L : Sense and Sensibilia, reconstructed from the manuscript notes by G.J. Warnock, Oxford University Press, « Oxford Paperbacks », Oxford, 1964 ; trad. fr. de P. Gochet, Le langage de la perception, Paris : Armand Colin, 1971.

Austin, J.L. : How to Do Things with Words (edited by J.O. Urmson and M. Sbisà), Oxford, Oxford University Press, 1975 ; trad. fr. G. Lane, Quand dire c'est faire, Paris, Seuil, 1970 ; réédité avec une postface de F. Récanati dans la coll. « Points-essais », 1991.

Baz, A. : When Words Are Called For, Cambridge, Mass. : Harvard University Press, 2012.

Benoist, J. (éd.) : Propositions et états de choses, Paris : Vrin, 2006.

Bonnet, V. : Aspects du sujet dans la philosophie du langage ordinaire, Thèse de doctorat de l'Université Bordeaux 3, 2012, 361 p.

Cappelen H. \& Hawthorne J. : Relativism and Monadic Truth, Oxford : Oxford University Press, 2009.

Davidson, D. : « A Coherence Theory of Truth and Knowledge », E. LePore (Ed.), Truth and Interpretations : Perspective on the Philosophy of Donald Davidson, Oxford : Blackwell, 1986, p. 309 ; trad. fr. de V. Aucouturier, «Une conception cohérentiste de la vérité et de la connaissance », in B. Ambroise \& S. Laugier (éd.), Textes-clés de Philosophie du langage, Vol 1, Paris : Vrin, 2009, pp. 305-338.

Frege, G. : Die Grundlagen der Arithmetik: eine logisch mathematische Untersuchung über den Begriff der Zahl, Hamburg : Meiner, 1986 ; trad. fr. de Cl. Imbert, Les Fondements de l'arithmétique : recherche logico-mathématique sur le concept de nombre, Paris : Seuil, 1969

Frege, G. : « Über Sinn und Bedeutung » Zeitschrift für Philosophie und philosophische Kritik, 100, 1892, p. 25-50 ; trad. fr. de J. Benoist, "Sur le sens et la référence », in B. Ambroise \& S. Laugier (éd.), Textes-clés de Philosophie du langage, Vol. 1, Paris : Vrin, 2009, pp. 49-84.

Frege, G. : « Der Gedanke », Beiträge zur Philosophie des deutschen Idealismus, 2, 1918-1919, p. 58-77 ; trad. Fr. de J. Benoist, « La pensée, une recherche logique », in B. Ambroise \& S. Laugier (éd.), Textes-clés de Philosophie du langage, Vol. 1, Paris : Vrin, 2009, pp. 85-124.

Kaplan, D. : « Demonstratives », in Almog, Perry \& Wettstein (Eds.), Themes from Kaplan, Oxford : Oxford University Press, 1989, pp. 481-563 ; trad. fr. de Ph. De Rouilhan et F. Rivenc, «Démonstratifs », in B. Ambroise \& S. Laugier (éd.), Textes-clés de Philosophie du langage, vol. 2, Paris : Vrin, 2011, pp. 135-181.

Katz, J. : The Metaphysics of Meaning, Cambridge, Mass. : MIT Press, 1990. 
Kukla, R. \& Lance, M. : 'Yo!' and 'Lo!', The Pragmatic Topography of the Space of Reasons, Cambridge, Mass. : Harvard University Press, 2009.

McDowell, J. : Meaning, knowledge, and reality, Cambridge, Mass. : Harvard University Press, 1998.

Narboux, J.-Ph. : « The logical fabric of assertions », in Ch. Al-Saleh \& S. Laugier (éd.), J.L. Austin et la philosophie du langage ordinaire, Hildesheim : Olms, pp. 183-232.

Narboux, J.-Ph. : «'There's many a Slip between Cup and Lip’: Dimension and Negation in Austin », in M. Gustafsson et R. Sørli, eds., The Philosophy of J. L. Austin, Oxford : Oxford University Press, 2011, pp. 204-239.

Récanati, F. : Perspectival Thought, A Plea for (Moderate) Relativism, Oxford : Oxford University Press, 2007.

Travis, Ch. : The True and the False, Amsterdam : J. Benjamins, 1981.

Travis, Ch. : « On What is Strictly Speaking True », Canadian Journal of Philosophy, n 15, 1985, pp. 187-229.

Travis, Ch. : The Uses of Sense : Wittgenstein's Philosophy of Language, Oxford : Oxford University Press, 1989.

Travis, Ch. : « Annals of Analysis », Mind, vol. 100, April 1991, pp. 237-264.

Travis, Ch. : « Order out of Messes », Mind, vol. 104, January 1995, pp. 133-144.

Travis, Ch. : « Meaning's Role in Truth », Mind, vol. 105, July 1996, pp. 451-466; trad. fr. de B. Ambroise \& V. Aucouturier, « Le rôle de la signification dans la vérité », », in B. Ambroise \& S. Laugier (Eds.), Textes-clés de Philosophie du langage, vol. 2, Paris : Vrin, 2011, pp. 181-210.

Travis, Ch. : «Pragmatics », in B. Hale \& C. Wright (Eds.), A Companion to the Philosophy of Language, Oxford : Blackwell, 1997, pp. 87-107

Travis, Ch. : « Taking Thought », Mind, vol. 109, July 2000, pp. 533-558.

Travis, Ch. : « Mind Dependence », Revue Internationale de Philosophie, $\mathrm{N}^{\circ} 218,4$ /2001, Paris : P.U.F., pp. 503-524.

Travis, Ch. : Unshadowed Thought, Cambridge, Mass. : Harvard University Press, 2001.

Travis, Ch. : « Frege's Target », Logic, Thought and Language (Royal Institute of Philosophy Lectures), Cambridge University Press, pp. 341-379, 2002.

Travis, Ch. : « Thought's Social Nature », ", European Journal of Philosophy, 2010, DOI: 10.1111/ j.1468-0378.2010.00402.x, pp. 1-22. ; trad. fr. B. Ambroise \& Ch. Gauvry, in B. Ambroise \& Ch. Chauviré (éd.), Le mental et le social, Editions de l'EHESS, coll. « Raisons Pratiques », 2013, pp. 37-65.

Travis, Ch. : Les liaisons ordinaires, Wittgenstein sur la pensée et le monde, Paris : Vrin, 2003 [version anglaise remaniée : Thought's Footing: A Themes in Wittgenstein's Philosophical Investigations, Oxford : Oxford University Press, 2006].

Travis, Ch. : Occasion-Sensitivity, Oxford : Oxford University Press, 2008.

Travis, Ch. : Objectivity and the Parochial, Oxford : Oxford University Press, 2010.

Wiggins, D. : « Meaning and truth conditions : from Frege's grand design to Davidson's », in B.

Hale \& C. Wright, eds., A Companion to the Philosophy of Language, Oxford : Blackwell, 1997, pp. 3-28.

Wittgenstein, L. : Tractatus Logico-philosophicus, London : Routledge \& Kegan Paul, 1922 ; trad. fr. G. Gaston-Granger, Tractatus logico-philosophicus, Paris : Gallimard, 1993. 
Wittgenstein, L. : Philosophical Investigations, translated by G.E.M. Anscombe, $3^{\text {rd }}$ ed., London :

Blackwell, 2001.

Wittgenstein, L. : Über Gewissheit, Oxford : Blackwell, 1969, 1975 ; trad. fr. De D. Moyal-Sharrock, De la certitude, Paris : Gallimard, 2006.

\section{NOTES}

1. Voir notamment J.L. Austin, "The Meaning of a Word» (1940) et "Truth" (1950), in Philosophical Papers, Oxford : Oxford University Press, 1979, pp. 55-75 et pp. 117-133.

2. Notamment L. Wittgenstein, Philosophical Investigations, translated by G.E.M. Anscombe, $3^{\text {rd }}$ ed., London : Blackwell, 2001 ; trad. fr. sous la dir. d'E. Rigal, Recherches philosophiques, Paris: Gallimard, 2004. Voir le commentaire qu'en a offert Travis : Ch. Travis, The Uses of Sense, Oxford : Oxford University Press, 1989.

3. Voir notamment Ch. Travis, "Pragmatics ", in B. Hale \& C. Wright (Eds.), A Companion to the Philosophy of Language, Oxford: Blackwell, 1997, pp. 87-107; reprinted in Ch. Travis, OccasionSensitivity, Oxford : Oxford University Press, 2008, pp. 109-129. Sur les rapports entre la conception contextualiste de Travis et la pragmatique, voir le texte de Ch. Gauvry dans ce même numéro.

4. Pour un exemple, voir D. Wiggins, « Meaning and truth conditions : from Frege's grand design to Davidson's », in B. Hale \& C. Wright, eds., A Companion to the Philosophy of Language, Oxford : Blackwell, 1997, pp. 3-28.

5. Tout dépend précisément de ce qu'on entend par «signification» et plus largement par "sémantique ». Voir Ch. Travis, «Pragmatics", art. cit. Voir aussi les remarques d'A. Baz, in When Words Are Called For, Cambridge, Mass. : Harvard University Press, 2012.

6. Voir notamment les deux textes principaux de Ch. Travis à cet égard: Unshadowed Thought, Cambridge, Mass. : Harvard University Press, 2001, et Les liaisons ordinaires, Wittgenstein sur la pensée et le monde, Paris : Vrin, 2003. C'est une reprise d'Austin, "The Meaning of a Word ", art. cit.

7. Précisons qu'il est devenu quasi-impensable, pour l'immense majorité de la philosophie analytique et pour une bonne part de la linguistique inspirée par elle, d'analyser la signification autrement qu'en fonction des valeurs de vérité supposées auxquelles elle renvoie.

8. Pour un examen détaillé de cette idée, défendue tant par Ch. Travis que par J.L. Austin, nous nous permettons de renvoyer à notre travail de thèse, B. Ambroise, Les pouvoirs du langage: la contribution de J.L. Austin à une conception contextualiste des actes de parole, Thèse de doctorat de l'Université Paris X - Nanterre, 2005. Cette idée est également défendue par R. Kukla et M. Lance, in 'Yo!' and 'Lo!', The Pragmatic Topography of the Space of Reasons, Cambridge, Mass. : Harvard University Press, 2009.

9. Notamment dans «Truth and Merit », repris in Ch. Travis, Objectivity and the Parochial, Oxford : Oxford University Press, 2011, pp. 229-262. En fait, on note dans le parcours de Travis un intérêt de plus en plus soutenu pour les idées de Frege et un usage désormais majeur de celles-ci.

10. Voir G. Frege, Die Grundlagen der Arithmetik: eine logisch mathematische Untersuchung über den Begriff der Zahl, Hamburg : Meiner, 1986 ; trad. fr. par Cl. Imbert, Les Fondements de l'arithmétique : recherche logico-mathématique sur le concept de nombre, Paris : Seuil, 1969, plus particulièrement la préface.

11. Ch. Travis, Unshadowed Thought, op. cit., p. 9.

12. Voir Ch. Travis, Unshadowed Thought, op. cit., p. 15.

13. Précisons immédiatement qu'il existe désormais une vaste littérature dite "contextualiste », mais que la position de $\mathrm{Ch}$. Travis s'en distingue par son caractère radical, qui tient à la 
conception pragmatique de la vérité qu'on va défendre dans la suite du texte. Sur cet éventail de positions contextualistes, voir encore A. Baz, When Words Are Called For, op. cit.

14. Pour être vraiment complète, ne serait-ce qu'historiquement, cette analyse devrait passer par "l'étape austinienne ", puisque la critique que $\mathrm{Ch}$. Travis opère du représentationnalisme doit beaucoup à la conception austinienne de la signification et de l'acte de parole. Faute de place, nous nous permettons de renvoyer, pour une présentation synthétique, à B. Ambroise, «J. L. Austin : de la philosophie du langage ordinaire à la conception de la parole comme action », in S. Laugier \& S. Plaud (éd.), La Philosophie analytique, Paris : Ellipses, 2011, pp. 179-196. On trouvera d'autres éléments plus précis dans la thèse de V. Bonnet, Aspects du sujet dans la philosophie du langage ordinaire, Thèse de doctorat de l'Université Bordeaux 3, 2012 ; et surtout dans J.-Ph. Narboux, "The logical fabric of assertions ", in Ch. Al-Saleh \& S. Laugier (éd.), J.L. Austin et la philosophie du langage ordinaire, Hildesheim : Olms, 2011, pp. 183-232.

15. Voir les présentations des deux textes de Frege, «Sur le sens et la référence » et "La pensée, une recherche logique », in B. Ambroise \& S. Laugier (éd.), Textes-clés de Philosophie du langage, Vol. 1, Paris : Vrin, 2009.

16. Voir G. Frege, «Über Sinn und Bedeutung » Zeitschrift für Philosophie und philosophische Kritik, 100, 1892, p. 25-50 ; trad. fr. de J. Benoist, "Sur le sens et la référence", in B. Ambroise \& S. Laugier (éd.), Textes-clés de Philosophie du langage, Vol. 1, Paris : Vrin, 2009, pp. 49-84.

17. G. Frege, « Sur le sens et la référence ", art. cit., p. 53.

18. Sur cette question, voir Ch. Travis, "Thought's Social Nature", », European Journal of Philosophy, 2010, DOI: 10.1111/j.1468-0378.2010.00402.x, pp. 1-22.; trad. fr. B. Ambroise \& Ch. Gauvry, in B. Ambroise \& Ch. Chauviré (éd.), Le mental et le social, Editions de l'EHESS, coll. «Raisons Pratiques», 2013, pp. 37-65.

19. Voir G. Frege, « Sur le sens et la référence », art. cit., p. 69.

20. G. Frege, «Sur le sens et la référence », art. cit. p. 64.

21. C'est pourquoi dans son idéographie, Frege ajoutera le signe d'assertion, dont la fonction est d'exprimer le jugement qu'une pensée est vraie, comme Austin ajoutera plus tard la force illocutoire au sens de l'énoncé. Voir D. Vernant, Du discours à l'action, Paris : PUF, 1997, et Discours et vérité, Paris : Vrin, 2009.

22. Voir encore D. Wiggins, «Meaning and truth conditions: from Frege's grand design to Davidson's », art. cit.

23. L. Wittgenstein, Tractatus Logico-philosophicus, London: Routledge \& Kegan Paul, 1922 ; trad. fr. G. Gaston-Granger, Tractatus logico-philosophicus, Paris : Gallimard, 1993. Précisons ici que nous proposons une lecture $\mathrm{du}$ " premier » Witgenstein assez répandue dans la philosophie analytique et qui n'est pas nécessairement conforme à la philosophie réellement exprimée par Wittgenstein dans le Tractatus.

24. Voir les exemples donnés par Travis in « Pragmatics», art. cit.

25. D. Davidson, "A Coherence Theory of Truth and Knowledge ", E. LePore (Ed.), Truth and Interpretations : Perspective on the Philosophy of Donald Davidson, Oxford : Blackwell, 1986, p. 309 ; trad. fr. inédite de V. Aucouturier, in B. Ambroise \& S. Laugier (éd.), Textes-clés de Philosophie du langage, Vol. 1, op. cit., 2009, pp. 305-337. On trouve en fait déjà une conception similaire chez Aristote : «Les sons émis par la voix sont les symboles des états de l'âme, et les mots écrits les symboles des mots émis par la voix. Et de même que l'écriture n'est pas la même chez tous les hommes, les mots parlés ne sont pas non plus les mêmes, bien que les états de l'âme dont ces expressions sont les signes immédiats soient identiques chez tous, comme sont identiques aussi les choses dont ces états sont les images. » (Aristote, De l'interprétation, 1, 16a 3-10)

26. Voir Unshadowed Thought, op. cit., p. 199.

27. Il s'agit ici d'un diagnostic : ce "platonisme linguistique » ne prend pas nécessairement la forme que lui donnait Frege lorsqu'il autonomisait la dimension du sens et de la pensée dans une 
volonté anti-psychologiste, et est même rarement revendiqué comme tel. Sur ces questions, voir J. Benoist (éd.), Propositions et états de choses, Paris : Vrin, 2006.

28. D. Kaplan, « Demonstratives », in Almog, Perry \& Wettstein (Eds.), Themes from Kaplan, Oxford : Oxford University Press, 1989, pp. 481-563 ; trad. fr. inédite de Ph. De Rouilhan et F. Rivenc, «Démonstratifs", in B. Ambroise \& S. Laugier (Eds.), Textes-clés de Philosophie du langage, vol. 2, Paris : Vrin, 2011, pp. 135-180.

29. Ch. Travis, "Pragmatics", art. cit., p. 104 : les ombres sont "des objets sémantiques interpolés entre les mots et l'état des choses qui rend les mots vrais ou faux, et d'une certain façon plus proches de cet état des choses que les mots eux-mêmes ".

30. Pour un examen des différentes façons dont la philosophie a, depuis Frege, pu poser ce niveau des «représentations" (ou un niveau équivalent), voir J. Benoist (éd.), Propositions et états de choses, op. cit.

31. Ch. Travis, Unshadowed Thought, op. cit., p. 203.

32. Ibid.

33. Ch. Travis, Unshadowed Thought, op. cit., p. 70.

34. Voir G. Frege, «Sur le sens et la référence », art. cit.

35. Voir Ch. Travis, Unshadowed Thought, op. cit., p. 65.

36. Ch. Travis, « Pragmatics", art. cit., p. 91.

37. Ce sont également les pratiques dans lesquelles s'inscrivent les énoncés qui permettent de déterminer si deux énoncés ont le même contenu représentationnel - cela se mesure notamment au niveau des conséquences tirées sur un plan pratique de leur énonciation. Si je réagis de la même façon à un énoncé $a$ et à un énoncé $b$, alors on peut considérer que, dans ce cas précis, ils ont le même contenu représentationnel, ils veulent dire la même chose.

38. Pour cette analyse, voir Ch. Travis, Unshadowed Thought, op. cit., pp. 90-93.

39. Sur cette question de la façon dont le contexte intervient, et sur le débat qui s'ensuit entre la "sémantique pragmatique » et le «contextualisme radical », voir encore le texte de Ch. Gauvry, dans ce numéro.

40. Pour un aperçu synthétique de cet argument, voir Ch. Travis, Occasion-Sensitivity, op. cit., «Introduction », p. 6.

41. . Ch. Travis, Unshadowed Thought, op. cit., p. 24.

42. Ibid, p. 210. On peut aussi considérer que Travis renvoit ici à la position philosophique de J. McDowell. Voir, par exemple, J. McDowell, Meaning, knowledge, and reality, Cambridge, Mass. : Harvard University Press, 1998.

43. Un premier aperçu s'en trouve dans Ch. Travis, "Pragmatics", art. cit. Une conception plus raffinée, mais aussi plus ardue à saisir, est exposée dans Ch. Travis, The True and the False, Amsterdam : J. Benjamins, 1981.

44. Ch. Travis, " Truth and Merit », art. cit., p. 258.

45. J. L. Austin, "Truth», art. cit. - ailleurs Austin se demandera si l'énoncé «La France est hexagonale » est vrai. Voir sur ce point J.-Ph. Narboux, "'There's many a Slip between Cup and Lip': Dimension and Negation in Austin ", in M. Gustafsson et R. Sørli, eds., The Philosophy of J. L. Austin, Oxford : Oxford University Press, 2011, pp. 204-239.

46. Ch. Travis, Unshadowed Thought, op. cit., p. 95.

47. Voir J.L. Austin, How to Do Things with Words (edited by J.O. Urmson and M. Sbisà), Oxford, Oxford University Press, 1975 ; trad. fr. G. Lane, Quand dire c'est faire, Paris, Seuil, 1970 ; réédité avec une postface de F. Récanati dans la coll. « Points-essais », 1991.

48. Dans un telle conception de la vérité, la non-vérité n'implique pas la fausseté, car ce sont deux dimensions d'évaluation qui s'inscrivent dans tout un continuum pouvant admettre des nuances. Voir, là encore, J.L. Austin, « Truth », art. cit., et Ch. Travis, « Truth and Merit ». Il peut aussi arriver que les circonstances ne permettent pas de déterminer si ce qu'on dit est vrai ou faux ; il faut alors reformuler, préciser les choses, etc. 
49. Ch. Travis, Les liaison ordinaires, op. cit., p. 202.

50. Ch. Travis, Les liaison ordinaires, op. cit., p. 195.

\section{RÉSUMÉS}

En philosophie du langage, une conception largement répandue veut que la signification d'une phrase soit analysable en fonction d'une proposition qui permet de spécifier les conditions de vérité de la phrase énoncée. La signification permettrait ainsi de déterminer immédiatement dans quelles conditions l'énonciation d'une phrase qui en est dotée est vraie. S'opposant rigoureusement à cette conception, $\mathrm{Ch}$. Travis entend découpler la signification des mots de leurs conditions de vérité pour montrer, au moyen d'une argumentation contextualiste, que la vérité n'est pas une propriété sémantique des énoncés, mais bien plutôt une dimension pragmatique d'évaluation des énoncés, qui s'opère en contexte. Ce faisant, il ne conteste pas tant la position frégéenne qu'il n'en reprend certains éléments pour les associer aux idées anti-platonistes de J.L. Austin.

In Philosophy of language nowadays it is generally agreed that the meaning of a sentence can be analyzed in terms of a proposition determining its truth-conditions. Meaning would thus allow us to determine straightforwardly the conditions in which the utterance of a sentence having that meaning would be true. By criticizing radically such an approach, $\mathrm{Ch}$. Travis means to delink the meaning of words from their truth-conditions in order to show, by means of contextualist arguments, that truth is not a semantic property, but rather a pragmatic dimension of the evaluation of utterances, which is contextually assessed. In that process, Travis does not so much contest the Fregean account but retains some of its aspects that he associates with the radical anti-Platonist ideas of J.L. Austin.

\section{INDEX}

Mots-clés : sens, contexte, pragmatique, vérité, conditions de vérité, représentation, jugement, Frege, Austin, Travis

Keywords : meaning, context, pragmatics, truth, truth-conditions, representation, judgement, Frege, Austin, Travis

\section{AUTEUR}

\section{BRUNO AMBROISE}

Université de Picardie Jules-Vernes, CURAPP-ESS (UMR 7319 UPJV/CNRS) 